\title{
Commentary on "Percutaneous tibial nerve stimulation maintenance therapy for overactive bladder in women: long-term success rates and adherence"
}

\author{
Abdelmageed Abdelrahman ${ }^{1}$ \\ Received: 7 May 2020 / Accepted: 20 May 2020 / Published online: 16 June 2020 \\ (C) The International Urogynecological Association 2020
}

This was a retrospective study of 141 women with overactive bladder (OAB) from January 2015 to August 2017 [1]. The authors had the following objectives:

1. To identify predictors of treatment success in women with OAB after 1 year of percutaneous tibial nerve stimulation (PTNS) maintenance therapy

2. Identify trends in success rates during that 1 year

3. Assess maintenance treatment adherence

The definition of success was based on Patient Global Impression-Improvement (PGI-I) score of 1 ("very much better") or 2 ("much better") or a PGI-I score of 1, 2, or 3 ("a little better").

Women received a standard of 12 weekly 30 -min sessions, followed by indefinite monthly maintenance sessions for responders. Maintenance treatment were given every 2 weeks for the first two treatments and then monthly thereafter. All the women included were provided the PGI-I questionnaire at their 12th weekly visit and at every maintenance treatment visit. The authors excluded those who had incomplete questionnaire data, had received onabotulinumtoxinA injections into their detrusor or pelvic floor muscles within 1 year of PTNS initiation, had a prior sacral neuromodulator placed (unless the device was confirmed to be turned off or removed), or had previously undergone a trial of PTNS therapy. At 1 year, 71 out of $141(53.2 \%)$ had discontinued treatment. $66.2 \%$ of women reported a PGI-I score of 1 or 2 and $92.3 \%$ reported a PGI-I score of 1,2 or 3 at 1 year. The authors stated that there were no clinical factors associated with treatment

Abdelmageed Abdelrahman

abdelmageed@hotmail.co.uk

1 Department of Urogynaecology, Liverpool Women's Hospital NHS Foundation Trust, Liverpool, UK success at 1 year. They also concluded that discontinuation rates of PTNS maintenance therapy at 1 year are high; therefore, long-term treatment may be feasible for only a minority of women with $\mathrm{OAB}$.

Strengths of the study include the large number of women undergoing maintenance treatments. The use of two different definitions of success also allows flexibility for the individual clinician to assess success rates, depending on which scoring of the PGI-I is used in their routine practice. Limitations of this study include the retrospective study design. Additionally, important outcome measures, such as urgency incontinence episodes, symptom severity, improvements in individual symptoms, pad count and pad weight, were not reliably collected. Other important outcomes, which may influence treatment adherence, including travel distance to the clinic and patient mobility, were also not collected.

\section{Compliance with ethical standards}

Conflicts of interest None.

\section{Reference}

1. Jung CE, Menefee SA, Diwadkar GB. Percutaneous tibial nerve stimulation maintenance therapy for overactive bladder in women: long-term success rates and adherence. Int Urogynecol J. 2020. https://doi.org/10.1007/s00192-020-04325-1.

Publisher's note Springer Nature remains neutral with regard to jurisdictional claims in published maps and institutional affiliations. 